\title{
Complexos de Metals de Transição em Química Fina e Medicinal ApLICAÇões na IndÚstria Farmacêutica
}

\author{
Mariette M. Pereira ${ }^{1,}{ }^{*}$ e Maria José S. M. Moreno ${ }^{2}$
}

\begin{abstract}
E ste artigo contextualiza a importância dos complexos de metais de transição nos domínios da Química Fina, Química Medicinal e Química Verde e exemplifica o interesse da sua utilização em hidrogenações e hidroformilações catalíticas homogéneas que integram processos de produção de fármacos. O contributo do Grupo de Catálise \& Química Fina da Universidade de Coimbra (C\&QF), no âmbito da utilização de complexos metálicos de Rh para optimizar e ampliar a aplicação destas mesmas reacções ao design e síntese de compostos com potencial actividade biológica, é também referido.
\end{abstract}

\section{Metais de Transição em Química Fina e Medicinal}

A legislação portuguesa estabelece que medicamento é "toda a substância ou associação de substâncias apresentada como possuindo propriedades curativas ou preventivas de doenças em seres humanos ou dos seus sintomas ou que possa ser utilizada ou administrada no ser humano com vista a estabelecer um diagnóstico médico ou, exercendo uma acção farmacológica, imunológica ou metabólica, a restaurar, corrigir ou modificar funções fisiológicas" [1].

A crescente aplicação de complexos de coordenação de metais de transição como fármacos revela o interesse que têm suscitado em Química Medicinal, cuja actuação se centra na "descoberta, design, identificação e preparação de compostos biologicamente activos, bem como do estudo do respectivo metabolismo e modo de acção" [2]. O trabalho desenvolvido por Alfred Werner (Prémio Nobel, 1913) na investigação de compostos contendo cobalto, cloro e amónia, bem como a descoberta, na década de 60 do século $X X$, da actividade an-

\footnotetext{
1,* Departamento de Química, Universidade de Coimbra, Rua Larga, 3004-535 Coimbra E-mail: mmpereira@qui.uc.pt

2 E-mail: mmpereira@qui.uc.pt Pólo das Ciências da Saúde, Azinhaga de Santa Comba, 3000-548 Coimbra
}

titumoral de um complexo de Pt (II) e sua consequente introdução na clínica, constituíram marcos importantes nesta área científica [3]. Para além de serem os agentes antitumorais mais usados, os complexos de metais de transição, designados por metalofármacos, apresentam um espectro de propriedades terapêuticas alargado, podendo ser utilizados como anti-inflamatórios, antiartríticos, antibacterianos, antifúngicos, antivirais, anticonvulsivantes e antidiabéticos (Figura 1). De facto, o contributo para a área da saúde de compostos contendo metais é tão significativo que, actualmente, constitui uma indústria que movimenta biliões de Euros [4, 5]. Mas os compostos de coordenação destes metais, não só contribuem directamente para o arsenal de medicamentos disponíveis para o tratamento de diversas patologias [6, 7], como também concorrem, enquanto catalisadores das mais distintas transformações químicas, para a síntese de fármacos orgânicos não isoláveis de fontes naturais, sendo igualmente importantes na duplicação sintética de outros que têm essa origem, bem como na semi-síntese de moléculas com propriedades terapêuticas mais favoráveis do que as que provêm de matérias-primas naturais.

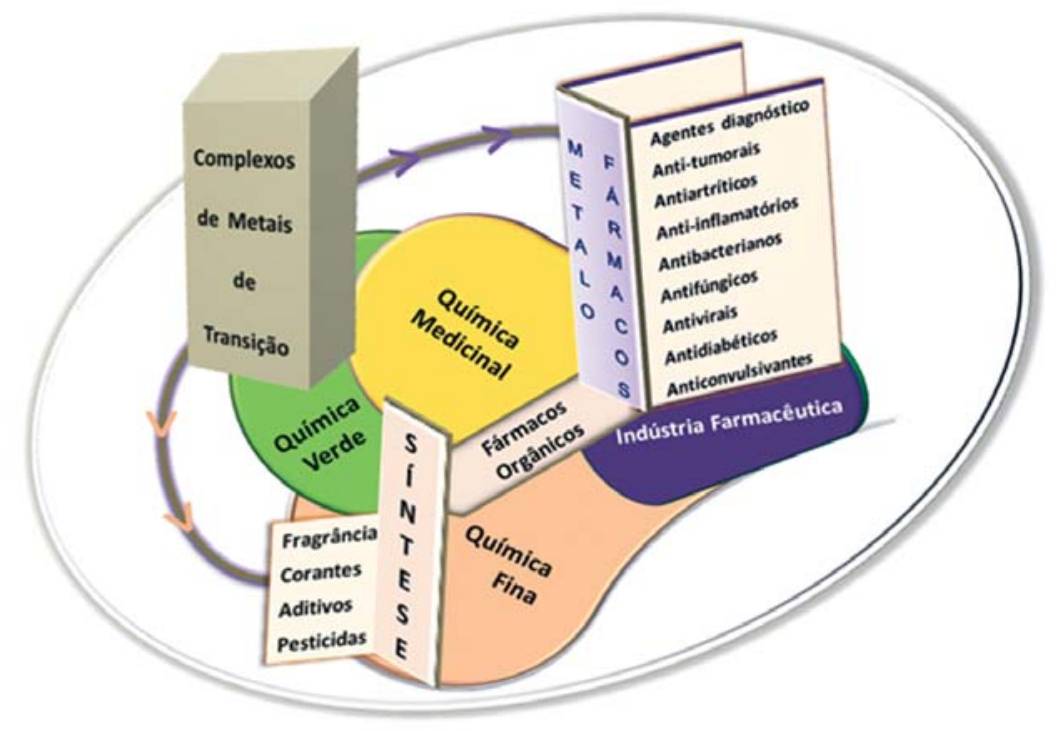

Figura 1 - Contextualização dos complexos de metais de transição como fármacos e catalisadores, no âmbito da Química Medicinal, Química Fina, Química Verde e Indústria Farmacêutica, evidenciando as inter-relações destes domínios 
Portanto, os complexos de metais de transição são da maior relevância para a Indústria Farmacêutica e, num senso mais lato, para a Química Fina, já que esta se ocupa da síntese de moléculas poli-funcionalizadas, com estereoquímica complexa e com utilização específica, onde se englobam também os fármacos, para além de pesticidas, fragrâncias, corantes e aditivos alimentares (Figura 1). Estes produtos, obtidos puros numa escala industrial que não excede as 10000 toneladas anuais, caracterizam-se por terem um elevado valor acrescentado [8].

Como exemplo ilustrativo desta realidade refere-se o fármaco mais utilizado em todo o mundo no tratamento de níveis elevados de colesterol, a atorvastatina (C33), cuja síntese se inicia com metanol (C1) e ácido acético (C2), ambos matérias-primas acessíveis que são manufacturadas em larga escala pela Química Pesada (Tabela 1). Após vários passos de preparação, originam os intermediários I e II que, tal como a atorvastatina, são considerados produtos de Química Fina. Nesta transformação há um elevado valor acrescentado que é ainda incrementado quando a atorvastatina é submetida ao processo subsequente de formulação, originando uma especialidade farmacêutica com um preço superior a $80000 \$ / \mathrm{kg}$ [8]. Outros exemplos que evidenciam bem o papel dos metais de transição na síntese de novos fármacos centramse na catálise com complexos de $\mathrm{Pd}$ na formação de ligações carbono-carbono, passo determinante na síntese de novas moléculas como a discodermolida [9], que possui propriedades imunossupressoras e antifúngicas e evidencia uma potencial actividade anticancerígena. A relevância da investigação realizada neste âmbito por R. F. Heck, A. Suzuki e E. Negishi levou a que fossem galardoados com o Prémio Nobel da Química 2010.

\section{Metais de Transição em Química Verde}

A produção industrial de fármacos e de outros produtos de Química Fina decorre tradicionalmente em múltiplos passos não catalíticos. A relação produto acabado/matéria-prima é menor do que para um produto proveniente da indústria química pesada e, devido à inerente complexidade estrutural destas moléculas, os processos de síntese são mais elaborados, o que significa maior produção de resíduos, incluindo subprodutos e co-produtos, solventes e reagentes usados, assim como resíduos de limpeza dos próprios equipamentos utilizados. O desafio actual, neste sector de produção de fármacos e seus intermediários de síntese é continuar a satisfazer as necessidades na área da saúde, fornecendo produtos seguros e eficazes, de uma forma economicamente viável e sem os efeitos colaterais adversos para o ambiente (Figura 1).

Estas novas exigências, longe de estarem atendidas, requerem a aplicação de estratégias e conceitos relacionados com desenvolvimento sustentável e química amiga do ambiente, também designada por Química Verde, que contempla a utilização de técnicas químicas e metodologias que promovem o design, desenvolvimento e implementação de processos de produção capazes de reduzir ou elimi- nar a formação de substâncias lesivas para a saúde e ambiente [10]. Neste contexto, é essencial o uso de catalisadores, matérias-primas renováveis, solventes alternativos/reacções sem solventes e a utilização sustentável de energia, entre outras abordagens inovadoras no âmbito da engenharia. Este conjunto de tecnologias ambientalmente benignas tem sido explorado e integrado em investigação e desenvolvimento de fármacos, para atingir a prevenção/redução de impactos ambientais na sua produção [11].

A título de exemplo salienta-se a síntese da sitagliptina, desenvolvida pela Merck em colaboração com a Solvias e que foi distinguida, em 2009, pela United States Environmental Protection Agency - EPA com O Greener Synthetic Pathways Award por incorporar princípios da Química Verde [12]. A obtenção deste fármaco, utilizado no tratamento da diabetes melitus tipo 2, inclui um passo sintético determinante que consiste na hidrogenação catalítica assimétrica de uma enamina intermediária desprotegida, utilizando como catalisador um complexo de Rh/t-Bu JOSIPHOS (Esquema 1).

Esta hidrogenação é altamente eficiente, permite o isolamento final da sitagliptina com elevada pureza óptica e química e reduz significativamente os resíduos gerados, com eliminação completa de efluentes aquosos [13]. Portanto, a adopção de medidas de prevenção em processos industriais, para além de promover um ganho ambiental significativo, apresenta-se como uma solução viável em termos económicos.

Tabela 1 - Exemplo da transformação de moléculas mono-funcionalizadas de Química Pesada em produtos poli-funcionalizados de Química Fina

\begin{tabular}{|c|c|c|c|c|}
\hline Parâmetros & $\begin{array}{c}\text { Metanol } \\
\mathrm{CH}_{4} \mathrm{O}\end{array}$ & $\begin{array}{l}\text { Ácido Acético } \\
\qquad \mathrm{C}_{2} \mathrm{H}_{4} \mathrm{O}_{2}\end{array}$ & \begin{tabular}{ccc}
\multicolumn{2}{c}{ Intermediários } \\
$\begin{array}{lll}\text { (I) } & \text { (II) } \\
\mathrm{C}_{7} \mathrm{H}_{11} \mathrm{NO}_{3} & \mathrm{C}_{14} \mathrm{H}_{30} \mathrm{NO}_{4}\end{array}$
\end{tabular} & $\begin{array}{l}\text { Atorvastatina } \\
\mathrm{C}_{33} \mathrm{H}_{35} \mathrm{FN}_{2} \mathrm{O}_{5}\end{array}$ \\
\hline Passos de preparação & 1 & 2 & 15 & 20 \\
\hline $\begin{array}{c}\text { Produção } \\
\text { (toneladas/ano) }\end{array}$ & $32 \times 10^{6}$ & $8 \times 10^{6}$ & 300 & 400 \\
\hline Preço $(\$ / k g)$ & 0.2 & 1.00 & 200 & 2000 \\
\hline
\end{tabular}




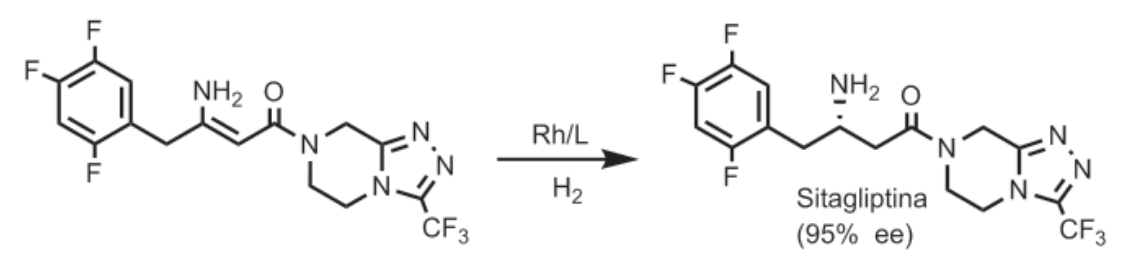

Esquema 1

Os métodos catalíticos podem efectivamente melhorar a economia atómica de uma reacção, que é um conceito fundamental em Química Verde [14], traduzindo-se em maximizar a conversão e selectividade, minimizando ou anulando a produção de subprodutos. Este papel dos catalisadores sai reforçado e enfatizado quando conseguem resumir uma sequência de várias etapas de síntese a uma conversão num único passo, reduzindo a energia necessária para o processo e diminuindo os resíduos produzidos. Portanto, também no âmbito da Química Verde, os complexos de metais de transição vêm dando um contributo substancial como catalisadores passíveis de serem usados em fase homogénea ou heterogénea.

Em regra, nos processos industriais, a catálise heterogénea é geralmente preferida porque facilita a recuperação do catalisador e permite um modo de operação contínua. No entanto, as temperaturas elevadas requeridas diminuem significativamente a selectividade dos processos. Em contrapartida, os sistemas catalíticos homogéneos possibilitam reacções mais rápidas e selectivas, para além de permitirem modelar o seu mecanismo, construindo catalisadores "por medida" através da variação de ligandos. De facto, o design adequado de um catalisador pode proporcionar uma melhoria significativa do seu desempenho numa transformação química específica [15].

Como a viabilização de reacções rápidas e com elevada quimio-, regio- e enantiosselectividade é de primordial peratura ambiente e pressão atmosférica. A hidrogenação de compostos multifuncionais tem sido e continua a ser objecto de investigação académica e aplicada, visando a concepção e desenvolvimento de catalisadores altamente activos e selectivos [16, 17]. Efectivamente, a evolução ao longo das últimas duas décadas da legislação que regulamenta a aprovação de medicamentos tem impulsionado a indústria farmacêutica a produzir, sistematicamente, fármacos quirais sintéticos na forma de compostos enantiomericamente puros, já que os dois enantiómeros podem causar efeitos biológicos diferentes.

importância na síntese industrial de fármacos quirais, os complexos de metais de transição cataliticamente activos, em fase homogénea, têm sido preferencialmente utilizados na sua produção, tal como se refere em seguida, até porque também são compatíveis com o tipo de processos comummente usados na indústria farmacêutica.

\section{Hidrogenações Catalíticas HOMOGÉNEAS}

A hidrogenação de olefinas é uma das reacções catalíticas mais extensamente estudadas, sendo os processos industriais maioritariamente heterogéneos. O primeiro sistema catalítico homogéneo a ser utilizado na hidrogenação de alcenos, alcinos e de aldeídos e cetonas foi o complexo $\mathrm{Rh}(\mathrm{PPh})_{3} \mathrm{Cl}$, comummente conhecido como catalisador de Wilkinson (Prémio Nobel da Química, 1973). Este catalisador permite a hidrogenação preferencial de alcenos não substituídos e de alcenos terminais, à tem-
A principal metodologia implementada com este objectivo envolve a catálise enantiosselectiva, permite transformar matérias-primas pró-quirais em moléculas quirais, com recurso a catalisadores adequados [18]. Pela investigação realizada neste âmbito, $\mathrm{W}$. Knowles e R. Noyori foram distinguidos, em 2001, com o Prémio Nobel da Química. Os sistemas catalíticos mais versáteis, utilizados em fase homogénea, são constituídos por complexos de metais de transição que integram ligandos quirais, o que lhes permite o reconhecimento da conformação preferencial do substrato insaturado. A primeira aplicação industrial relevante desta metodologia consistiu na produção da L-Dopa - fármaco quiral usado para o tratamento sintomático da doença de Parkinson - através da hidrogenação assimétrica dos derivados do ácido cinâmico (enamidas pró-quirais) com um complexo catiónico de Rh, contendo a difosfina quiral DIPAMP como ligando (Esquema 2) [19].

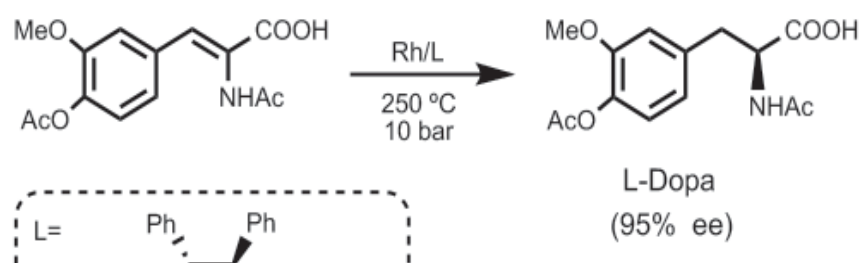

Esquema 2 
A actividade e a selectividade conseguidas nestas reacções catalíticas, para além de dependerem da estrutura da olefina, são também condicionadas pelo metal e ligando de fósforo utilizado. A experiência tem mostrado que, para o mesmo metal, a variação da estrutura do ligando pode permitir o ajuste das propriedades electrónicas e estéreas dos complexos cataliticamente activos, melhorando o resultado de reacções catalíticas, especialmente em termos da sua conversão, selectividade e impacto ambiental. De facto, a exploração do efeito dos ligandos nas propriedades dos complexos metálicos tem levado ao desenvolvimento de uma pletora de catalisadores homogéneos, úteis na produção de uma ampla gama de compostos [20].

A hidrogenação catalítica homogénea de compostos carbonílicos não está tão difundida. Para este efeito, os catalisadores de Ru (II) são mais activos que os análogos de Rh (I). Na hidrogenação industrial de aldeídos e cetonas contendo outros grupos polares como aminoácidos, hidroxilo, éster, amida e sulfato, que permitem uma interação adicional com o complexo metálico, têm sido obtidos elevados excessos enantioméricos [21].

Após a Monsanto ter introduzido a hidrogenação enantiosselectiva no processo de síntese da L-Dopa (Esquema 2), outras indústrias farmacêuticas adoptaram metodologias análogas na preparação de intermediários de outros fármacos, encontrando-se umas ainda em fase piloto e outras já plenamente implementadas na sua produção. Como exemplos representativos da hidrogenação catalítica enantiosselectiva de ligações $\mathrm{C}=\mathrm{C}$, refere-se a preparação de intermediários na síntese da vitamina E (Takasago), do inibidor da protease do HIV cilazapril (Roche) e do anti-hipertensor candoxatril (Chiroteck/Pfizer). A hidrogenação catalítica de ligações $\mathrm{C}=\mathrm{O}$ tem sido também aplicada no processo de síntese do anti-depressivo levoprotiline (Ciba-Geigi/Solvias) e do antibiótico carbapenemo (Takasago) (Esquema 3) [21].

Neste âmbito da hidrogenação, o grupo C\&QF investigou a aplicação do

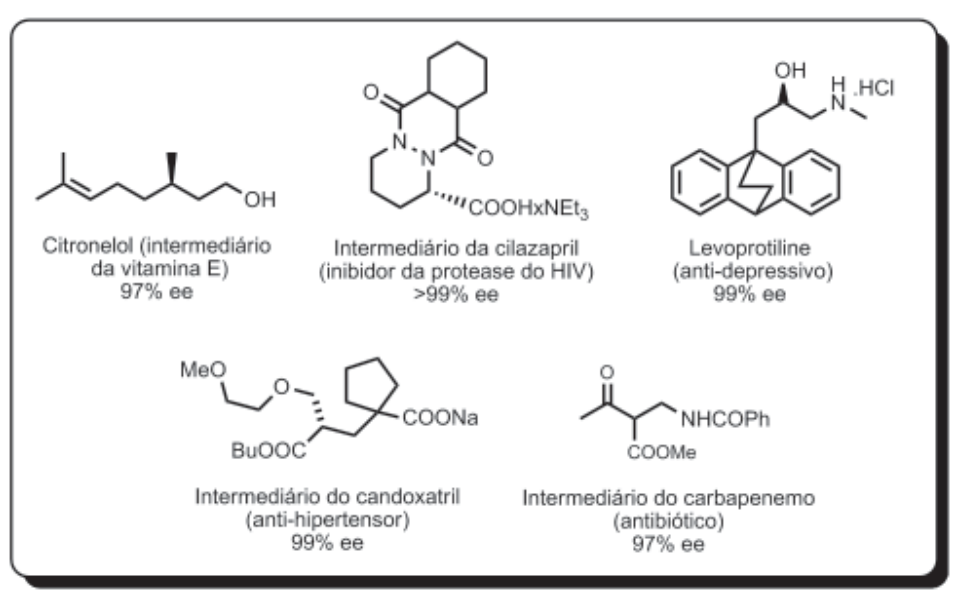

Esquema 3

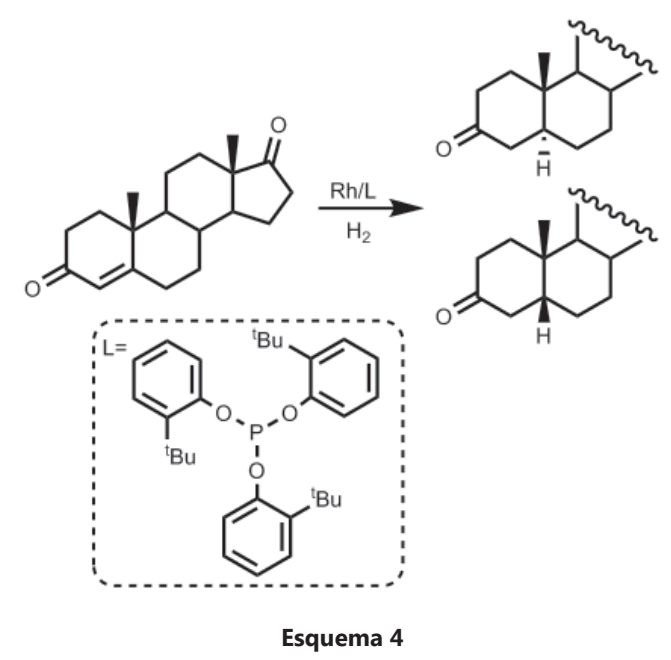

sistema catalítico de $\mathrm{Rh}(\mathrm{I})$ na hidrogenação de 3-oxo-esteróides insaturados em C-4, das séries androstano, colestano e pregnano, procedendo à variação sistemática de ligandos de fósforo no catalisador preparado in situ. Para a mesma reacção procedeuse ainda à modelação dos parâmetros razão substrato/catalisador, solvente, temperatura e pressão. Desta forma, foi possível obter as correspondentes cetonas saturadas com a desejável quimio- e estereosselectividade, situando-se os rendimentos na ordem dos 80\% (Esquema 4) [22]. Para este tipo de substratos, o isómero $5 \alpha-\mathrm{H}$, termodinamicamente mais estável, predomina nas reacções efectuadas com sistemas catalíticos homogéneos, enquanto o isómero $5 \beta-\mathrm{H}$ é o principal produto resultante do processo catalítico heterogéneo. Esta inversão de estereosselectividade tem merecido particular atenção pelo potencial que apresenta na síntese de hormonas e vitaminas.
Porém, a difícil recuperação do catalisador na hidrogenação catalítica homogénea destes oxo-esteróides $\alpha, \beta$-insaturados condicionava o seu potencial interesse na indústria farmacêutica. Para superar esta limitação efectuou-se a ligação do catalisador homogéneo a um suporte sólido adequado, de forma a viabilizar a sua heterogeneização. Assim, através da imobilização do catalisador em resinas de troca iónica ou num suporte sólido de $\mathrm{PtO}_{2}$, foi possível conseguir a sua reutilização, sem comprometimento das selectividades evidenciadas em fase homogénea, o que poderá vir a viabilizar, num futuro próximo, a transposição desta heterogeneização do catalisador para uma escala preparativa $[23,24]$.

Esta metodologia de heterogeneização de catalisadores homogéneos, para além de ter interesse na indústria, vem sendo objecto de investigação centrada no estudo de novas es- 
pécies catalíticas que possam revelar propriedades inusitadas.

\section{Hidrofomilações Catalíticas HoMOGÉNEAS}

A reacção de hidroformilação foi descoberta, acidentalmente, por Otto-Roelen em 1938 quando trabalhava no desenvolvimento da reacção de Fischer-Tropsch para a indústria Ruchermie [25]. Esta reacção consiste na adição de monóxido de carbono e hidrogénio a olefinas para formar o correspondente aldeído, num processo que pode ser catalisado por diferentes complexos de metais de transição (Esquema 5). De entre os diversos tipos de catalisadores aplicados a nível industrial, salientamos os complexos de cobalto e de ródio coordenados, ou não, com ligandos de fósforo [26]. Este processo catalítico permite formar novas ligações carbono-carbono com incorporação de todos os átomos dos reagentes $\left(\mathrm{H}_{2}\right.$ e $\left.\mathrm{CO}\right)$ no produto final, sendo por isso um excelente exemplo duma transformação com completa economia atómica. Apesar de nos primeiros tempos esta reacção ter sido fundamentalmente aplicada à síntese de produtos pouco funcionalizados do foro da Química Pesada, com o desenvolvimento de novos catalisadores quirais (Esquema 5) passou a ser também um processo relevante para Química Fina [27-30], nomeadamente na síntese industrial de um precursor da vitamina A (BASF e Hoffmann-La Roche) [31, 32]. A sua aplicação na produção de aldeídos quirais foi, durante muitos anos, limitada por restrições resultantes das baixas regio- e sobretudo enantiosselectividades dos processos catalíticos. Contudo, foram abertos novos horizontes com a emergência de ligandos quirais que permitem a aplicação desta reacção na preparação de aldeídos enantiomericamente puros, que são importantes building blocks/precursores de moléculas mais complexas, farmacologicamente activas, tais como: antibióticos, peptídeos, macrociclos antitumorais e prostaglandinas [30].

Devido ao interesse crescente na obtenção de novos derivados de ácidos aril-propiónicos quirais, com diversas aplicações na indústria farmacêutica para a preparação de substâncias

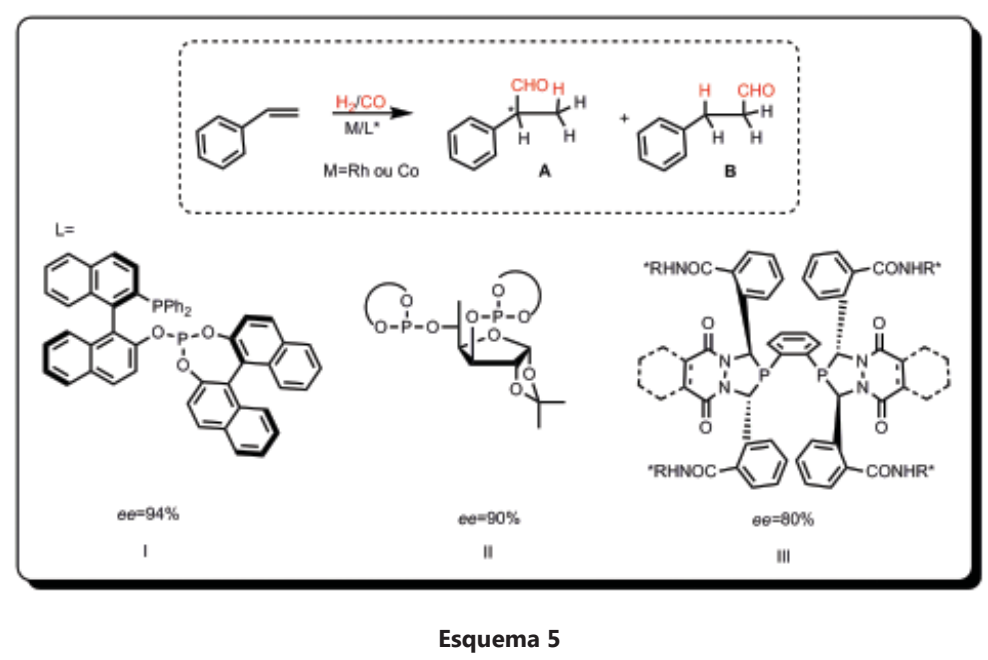

activas com reconhecida actividade inflamatória, o estireno tem sido um dos substratos modelo mais estudados nesta reacção [26]. No entanto, para que esta possa ser aplicada a nível industrial na preparação de ácidos aril-propiónicos [33] é importante desenvolver, não só, novos ligandos quirais/catalisadores, mas também optimizar as condições de reacção para obter elevadas regiosselectividades do aldeído ramificado (Esquema $5 A)$. Neste sentido, o grupo C\&QF desenvolveu recentemente estudos de optimização da regiosselectividade da hidroformilação de vinil-aromáticos como o estireno e a clorofila, recorrendo a um planeamento factorial $2^{2}$, o que permitiu atingir regiosselectividades próximas de $100 \%$ para o aldeído ramificado do estireno e de beta vinil-porfirinas, efectuando um número reduzido de experiências (Tabela 2). De facto, o planeamento factorial tem-se revelado como uma excelente ferramenta para a optimização de processos sintéticos [21, 34]

A utilização de complexos organometálicos na funcionalização de esteróides foi recentemente descrito por Kollar [35] num artigo de revisão, onde fica patente que a hidroformilação também permite introduzir um grupo formilo, num único passo e de forma diasterosselectiva, em duplas ligações internas de núcleos de esteróides. A primeira hidroformilação deste tipo de substratos foi descrita por Pike e Wender [36], nos anos 50 , utilizando como catalisador o complexo $\left[\mathrm{CoH}(\mathrm{CO})_{4}\right]$ em quantidades estequiométricas e recorrendo a condições de reacção muito severas.
Mais recentemente, surgiram outros exemplos da sua aplicação na funcionalização de esteróides insaturados em C-16, utilizando catalisadores de ródio coordenados com fosfinas alquílicas [37]. A contribuição do grupo $C \& Q F$, neste âmbito, traduziu-se no desenvolvimento da hidroformilação diastereosselectiva de esteróides de diversas séries, insaturados em C-4, utilizando complexos de ródio/tris(otert-butilfenil)fosfito como catalisador (Tabela 2) [38]. Esta reacção tem constituído um exemplo de referência, também a nível pedagógico, já que permite demonstrar experimentalmente a modulação da reactividade e da regio- e diastereosselectividade destas reacções catalíticas. Salientase ainda que a hidroformilação aplicada a terpenos naturais permite obter aldeídos que podem desempenhar um importante papel como intermediários em síntese de produtos do foro da Química Fina [39].

Neste domínio e no âmbito de um projecto Ibero-Americano que contempla a hidroformilação diastereosselectiva de produtos naturais, o grupo C\&QF desenvolveu estudos de hidroformilação de mono e diterpenos tendo em vista a obtenção de produtos de elevado valor acrescentado, nomeadamente derivados do acetato de mirtenol [38] (Tabela 2), assim como do ácido kaurénico e do kaurenol (Tabela 2), que é uma matéria-prima natural obtida a partir de uma planta resinosa "frailejón" nativa das montanhas dos Andes [41].

Os exemplos aqui apresentados evidenciam que o desenvolvimento 
recente de catalisadores quirais altamente regio e enantiosselectivos pode ampliar a utilização da hidroformilação como ferramenta sintética na obtenção de produtos de elevado valor acrescentado, designadamente com potenciais aplicações no domínio da Química Fina.

\section{Conclusão}

A indústria farmacêutica precisa de continuar a desenvolver e produzir complexos de metais de transição que possuam uma acção terapêutica inerente. Em simultâneo, carece também de novos complexos de metais de transição capazes de actuarem como catalisadores selectivos, recuperáveis e reutilizáveis, que permitam sintetizar novos fármacos ou optimizar a produção de outros já introduzidos na prática clínica e usados rotineiramente. Estas necessidades, compaginadas com estratégias e conceitos relacionados com Desenvolvimento Sustentável e Química Verde vêm liderando muita da investigação realizada no âmbito da Química Fina e Medicinal, no que se refere ao design, desenvolvimento e produção de compostos biologicamente activos. É nesta con- fluência inter- e transdisciplinar de interesses e objectivos que os complexos de metais de transição têm um papel relevante a protagonizar.

\section{Agradecimentos}

As autoras agradecem a contribuição das equipas de investigação dos Projectos PTDC/QUI/88015/2006 e PTDC/QUI/112913/2009 financiados pela Fundação para a Ciência e Tecnologia (FCT); do Projecto IberoAmericano CYTED-V-9 e dos alunos do Programa de Pós-Graduação do Grupo de Catálise \& Química Fina do Departamento de Química - Universidade de Coimbra, em particular A. F. Peixoto, A. R. Almeida, A. R. Abreu, R. Nunes e R. Carrilho.

\section{REFERÊNCIAS}

[1] Decreto-Lei $n^{\circ} 176 / 2006$ de 30 de Agosto; Artigo 30; alínea ee)

[2] C. G. Wermuth, C. R. Ganellin, P. Lindberg, L. A. Mitscher IUPAC, Pure and Applied Chemistry 70 (1998) 1129-1143.

[3] E. R. Jamieson, S. J. Lippard, Chem. Rev. 99 (1999) 2467-2498.

Tabela 2 - Hidroformilações Catalíticas

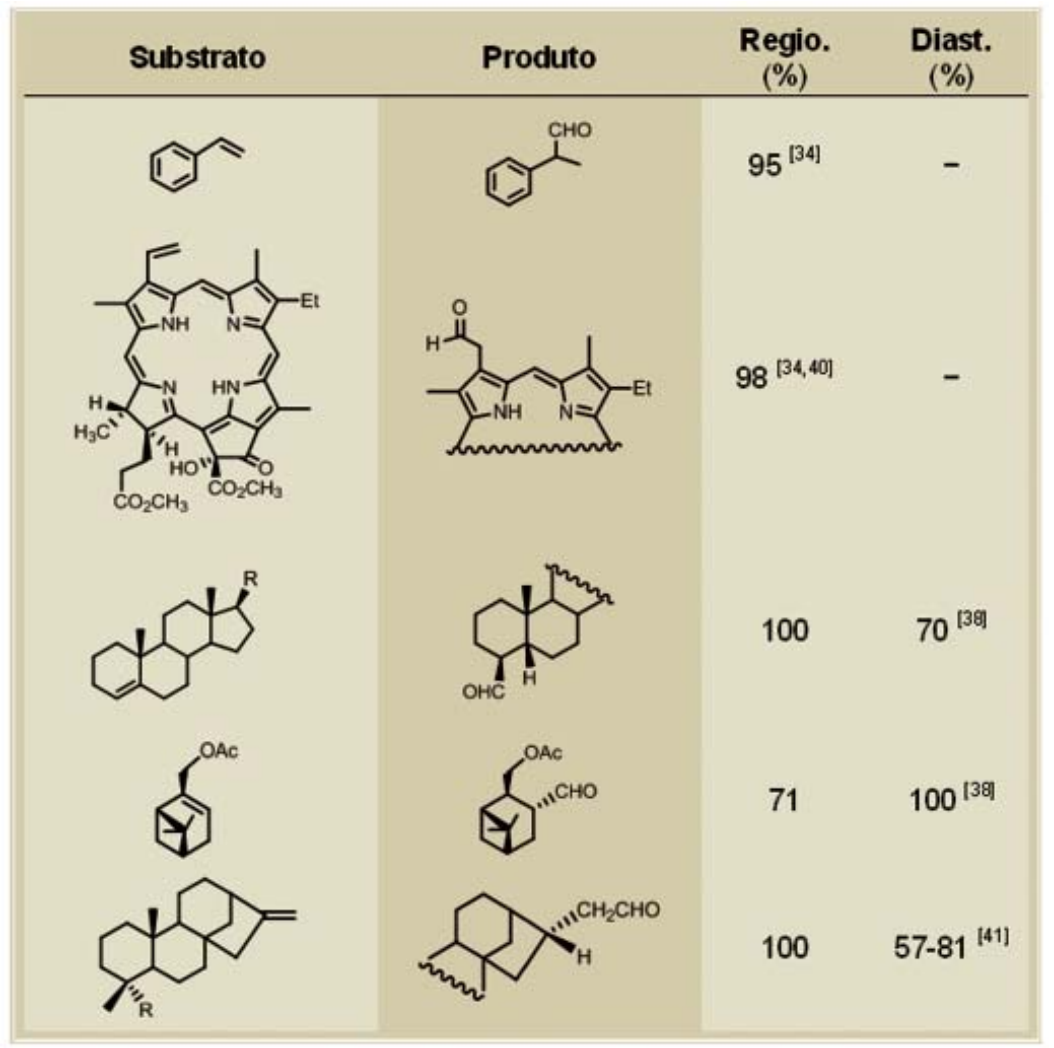

[4] D. Chen, V. Milacic, M. Frezza, Q. P. Dou, Curr. Pharm. Des. 15 (2009) 777-791.

[5] S. M. Cohen, Curr. Opin. Chem. Biol. 11 (2007) 115-120.

[6] S. P. Fricker, Dalton Trans. (2007) 4903-4917.

[7] S. Rafique, M. Idrees, A. Nasim, H. Akbar, A. Athar, Biotechnology and Molecular Biology Reviews 5 (2010) 38-45.

[8] P. Pollak, Fine Chemicals:The Industry and the Business, John Wiley \& Sons, 2007.

[9] S. P. Gunasekera, M. Gunasekera, R. E. Longley, G. K. Schulte, J. Org. Chem. 55 (1990) 4912-4915.

[10] P. T. Anastas, J. C. Warner, Química Verde: Teoria e Prática, Oxford University Press: New York, 1998.

[11] P. J. Dunn, A. S. Wells, M. T. Williams, Future Trends for Green Chemistry in the Pharmaceutical Industry. In Green Chemistry in the Pharmaceutical Industry (eds P. J. Dunn, A. S. Wells and M. T. Williams), Wiley-VCH Verlag $\mathrm{GmbH} \&$ Co: KGaA, Weinheim, Germany, 2010, cap. 16.

[12] http://www.epa.gov/gcc/pubs/docs/ award_recipients_1996_2010.pdf

[13] K. B. Hansen, Y. Hsiao, F. Xu, N. Rivera, A. Clausen, M. Kubryk, S. Krska, T. Rosner, B. Simmons, J. Balsells, N. Ikemoto, Y. Sun, F. Spindler, C. Malan, E. J. J. Grabowski, J. D. Armstrong, J. Am. Chem. Soc. 131 (2009) 87988804.

[14] B. M. Trost, Science 254 (1991) 14711477.

[15] P.W.N.M.vanLeeuwen, Homogeneous Catalysis. Understanding the Art, Kluwer Academic Publ.: Dordrecht, 2004.

[16] Comprehensive Asymmetric Catalysis-I in E. N. Jacobsen, A. Pfaltz, H. Yamamoto (eds.) Springer, Berlin-Heidelberg, 1999, vol. I, Cap. 5, pág. 102.

[17] R. H. Morris, Chem. Soc Rev. 38 (2009) 2282-2291.

[18] H. U. Blaser, Chem. Comm. (2003) 293-296.

[19] W. S. Knowles, Adv. Synth. Catal. 345 (2003) 3-13.

[20] Principles and Applications of Asymmetric Synthesis in G.-Q. Lin, Y.-M. Li, A.S.C.Chan(eds.)Wiley Inter-science, Great Britain, 2000, Cap.6, pág. 331.

[21] Catalysis from Theory to Application: An Integrated Course, in J. L. Figueiredo, M. M. Pereira, J. Faria (eds.), Coimbra, 2008.

[22] R. M. D. Nunes, A. F. Peixoto, M. R. Axet, M. M. Pereira, M. J. Moreno, L. 
Kollár, C. Claver, S. Castillón, J. Mol. Catal. A: Chem. 247 (2006) 275-282.

[23] R. M. D. Nunes, T. F. Fernandes, G. A. Carvalho, E. N. Santos, M. J. S. M. Moreno, A. P. Piedade, M. M. Pereira, J. Mol. Catal. A: Chem. 307 (2009) 115-120.

[24] R. M. D. Nunes, B. F. Machado, M. M. Pereira, M. J. S. M. Moreno, J. L. Faria, J. Mol. Catal. A: Chem, aceite.

[25] B. Cornils, W. A. Herrmann, M. Rasch, Angew. Chem. Int. Ed., 33 (1994) 2144-2163.

[26] P.W.N.M. van Leeuwen, C. Claver, Rhodium Catalyzed Hydroformylation, Kluwer Academic, Dordrecht, 2000.

[27] M. Beller, C. Bolm, Transition Metals for Organic Synthesis. Building Blocks and Fine Chemicals, Wiley-VCH, Weinheim,1998, pág. 25.

[28] A. Gual, C. Godard, S. Castillón, C.
Claver, Adv. Synth. Catal. 352 (2010) 463-477.

[29] J. Klosin, C. R. Landis, Acc. Chem. Res. 40 (2007) 1251-1259.

[30] R. V. Chaudhari, Curr. Opin. Drug Discov. 11 (2008) 820-828.

[31] G. W. Parshall, W. A. Nugent, Chem. Tech. 18 (1988) 184-190.

[32] P. Fitton, H. Moffet, U.S. Patent, US4124619-A, 1978.

[33] C. Botteghi, M. Marchetti, G. Ponte, Química Nova 20 (1997), 30.

[34] A. F. Peixoto, M. M. Pereira, A. A. C. C. Pais, J. Mol. Cat. A:Chem 267 (2007) 234-240.

[35] R. Skode-Folkes, L. Kollár, Chem Rev. 103 (2003) 4095-4129.

[36] a) P. F. Beal, M. A. Rebenstorf, J. E. Pike, J. Am. Chem. Soc. 81 (1959) 1231-1234; b) A. L. Nussbaum, T. L. Popper, E. P. Oliveto, S. Friedman, I.
Wender, J. Am. Chem. Soc. 81 (1959) 1228-1231.

[37] S. Toros, I. G.-Pécsi, B. Heil, S. Maho, Z. Tuba, J. Chem. Soc., Chem. Commun. (1992) 858-859.

[38] A. F. Peixoto, M. M. Pereira, A.M.S. Silva, C. M. Foca, J. C. Bayón, M. J. S. M. Moreno, A. M. Beja, J. A. Paixão, M. Ramos Silva, J. Mol. Cat. A:Chem 275 (2007) 121-129.

[39] J. L. F. Monteiro, C. O. Veloso, Topics in Catalysis 27 (2004) 169-180.

[40] A. F. Peixoto, M. M. Pereira, A. F. Sousa, A. A. C. C. Pais, M. G. P. M. S. Neves, A. M. S. Silva, J. A. S. Cavaleiro, J. Mol. Cat. A:Chem 235 (2005) 185-193.

[41] A. F. Peixoto, D. S. de Melo, T. F. Fernandes, Y. Fonseca, E. V. Gusevskaya, A. M.S. Silva, R. R. Contreras, M. Reyes, A. Usubillaga, E. N. dos Santos, M. M. Pereira, J. C. Bayón, Appl Cat. A: Gen. 340 (2008) 212-219.

\section{Actualidades Científicas}

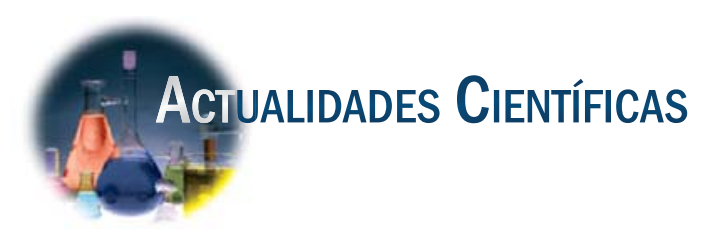

\section{Catalisadores Comutáveis}

Os catalisadores não estão normalmente equipados com um interruptor on-off. No entanto, segundo investigadores da Northwestern University, é possível que complexos supramoleculares cataliticamente activos possam ser desenhados de modo a transitar, reversivelmente e de uma forma controlável, entre configurações distintas capazes de, selectivamente, mediar ou inibir uma reacção (Science 2010, $330,66)$. Estes estudos sugerem novas estratégias para o projecto de catalisadores flexíveis, reguláveis através de meios químicos triviais.

Em resposta a um estímulo químico específico, como a presença de certos iões, enzimas alostéricas sofrem alterações conformacionais que possibilitam a regulação da actividade enzimática. Estas alterações são tipicamente desencadeadas pela ligação de um ião ou outra espécie química, a uma localização que não seja o centro activo da enzima.

Se este tipo de comportamento for extensível a substâncias organometálicas, então o alosterismo pode funcio- nar como uma nova abordagem para o controlo de reacções catalisadas por uma vasta gama de compostos. No entanto, a engenharia molecular relacionada com este tipo de mimetização da Natureza tem-se revelado deveras problemática.

A equipa da Northwestern que inclui os químicos Hyo Jae Yoon, Junpei Kuwabara, Jun-Hyun Kim e Chad A. Mirkin, recorreu a métodos de síntese supramolecular de forma a ultrapassar estes desafios. Os investigadores sintetizaram estruturas supramoleculares a partir de duas unidades inertes contendo ciclos multi-fenil e uma unidade cataliticamente activa que possui um ligando de $\mathrm{Al}(\mathrm{III})$-salen. As secções de multi-ciclos actuam como camadas de bloqueio ou abas que, dependendo da conformação especificada, podem expor ou ocultar o centro metálico, que actua como um catalisador de polimerização. Assim, a equipa demonstrou que a estrutura de três camadas pode mediar reacções de polimerização por abertura de anel de $\varepsilon$-caprolactona. O tratamento da forma fechada ou inactiva da estrutura com iões cloreto induz a abertura das abas, expondo o centro catalítico e despoletando a polimerização. Por outro lado, a adição de uma reduzida quantidade de um agente de remoção do cloreto causa o encerramento da estrutura e a consequente terminação da polimerização. Através da manipulação deste processo reversível, a equipa conseguiu sintonizar os pesos moleculares dos polímeros sintetizados.

"Este é um belo exemplo da forma como a flexibilidade da química supramolecular pode ser usada para mimetizar a Natureza", afirma o professor de química Wenbin Lin da University of North Carolina, em Chapel Hill, que acrescenta, "esta abordagem permite antecipar muitas aplicações interessantes".

(Adaptado do artigo de 4/10/2010 de Mitch Jacoby: Switchable

Catalysts, Chemical \& Engineering News, http://pubs.acs.org/cen/news/88/ i40/8840notw6.html)

PB 


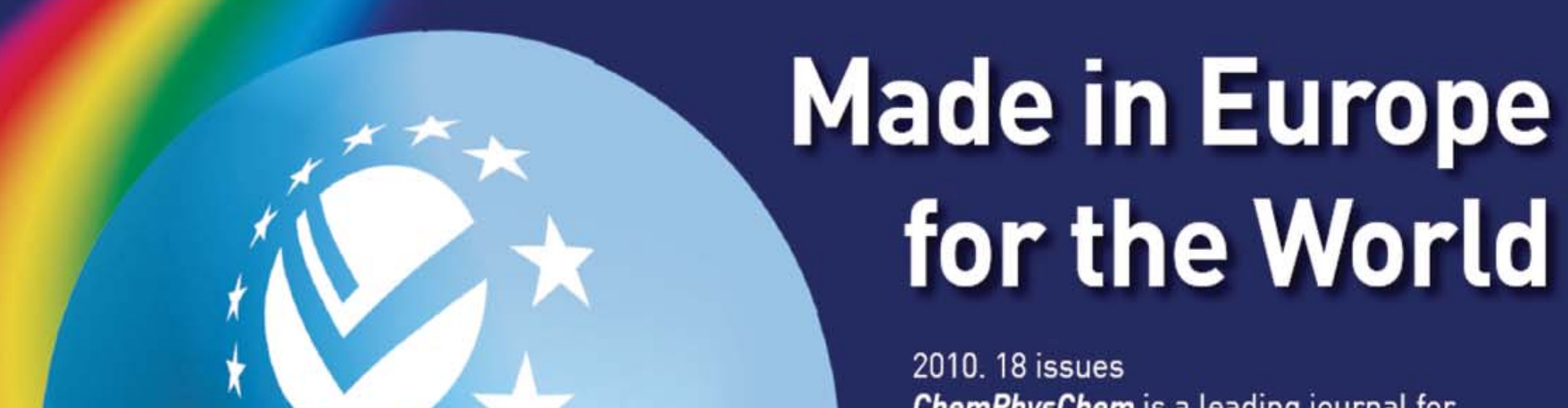

ChemPhysChem is a leading journal for chemical physics and physical chemistry. The journal offers an attractive mixture of Communications, Articles \& Minireviews www.chemphyschem.org

\section{ChemPubSoc}

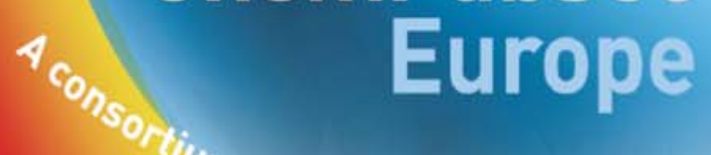

2010. 18 Issues.

ChemBioChem is a leading journal for chemical biology and biological chemistry. The journal offers an attractive mixture of Communications, Full Papers, Reviews \& Minireviews www.chembiochem.org

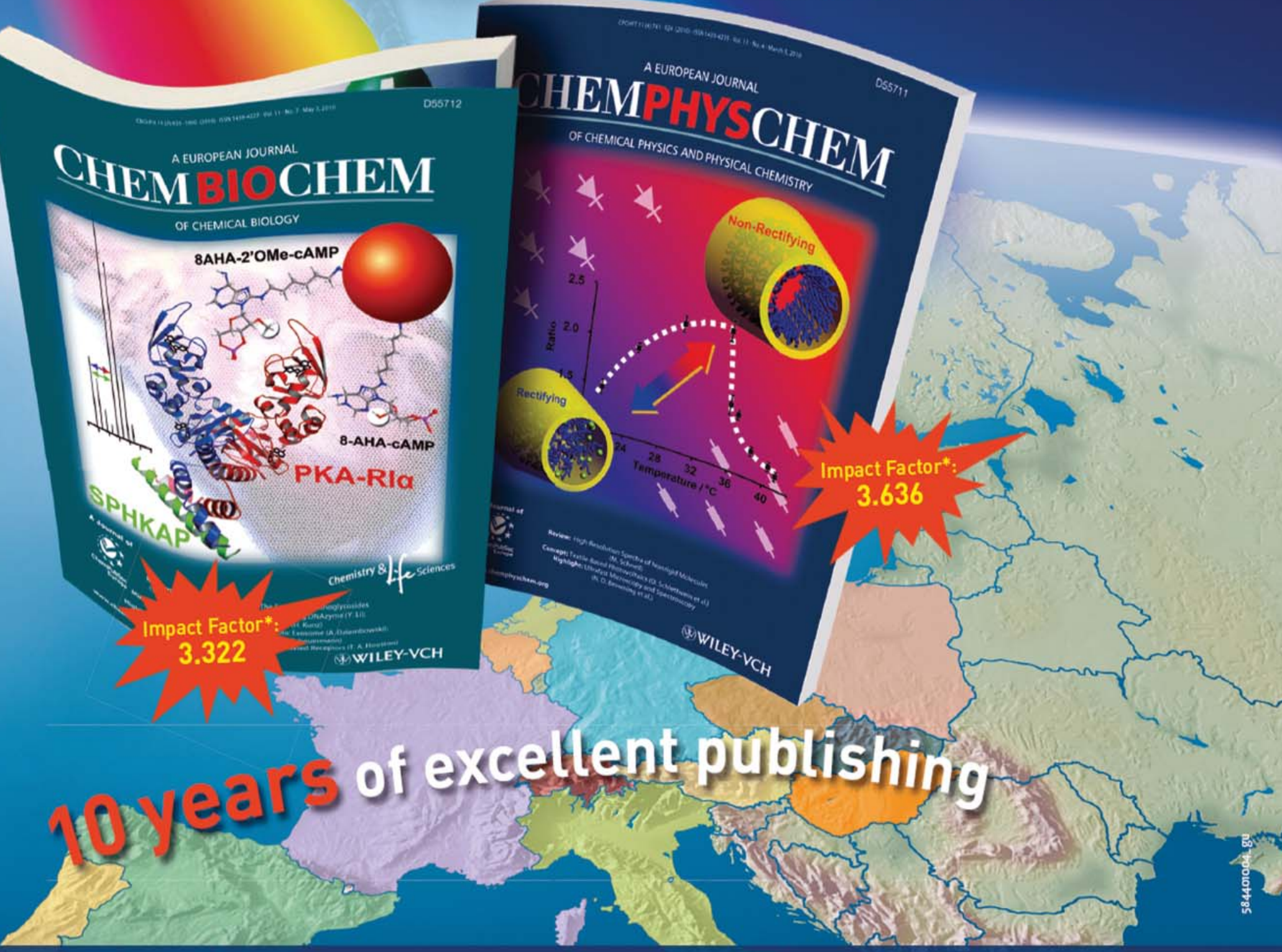

*2008 Journal Citation Reports ${ }^{\oplus}$ (Thomson Reuters, 2009]

For further information and to subscribe please send an e-mail to:

cs-journalsdawiley.com

(Americas, Europe, Middle East and Africa, Asia Pacific) 